\title{
The perceived feasibility and acceptability of a plant-based low carbohydrate diet for the management and prevention of type 2 diabetes
}

\author{
H.R Molloy, K.G. Jackson and M.E Clegg \\ Hugh Sinclair Unit of Human Nutrition, Department of Food and Nutritional Sciences, University of Reading, \\ Reading, RG6 6AP
}

The increasing global prevalence of type 2 diabetes mellitus (T2DM) means there is a clear need for prevention strategies focussing on the diet and lifestyle factors of high-risk individuals ${ }^{(1)}$. Low carbohydrate (LC) diets $(<130 \mathrm{~g}$ carbohydrates/day) and ketogenic diets $(<50 \mathrm{~g}$ carbohydrates/day) have become increasingly popular in a clinical setting for managing T2DM, however, there are concerns around their elevated saturated fat content ${ }^{(2)}$. There is now emerging evidence to suggest cardiometabolic benefits of plant-based diets which are typically lower in saturated fat, and higher in dietary fibre, unsaturated fat, and polyphenols, all of which have shown protective effects against $\mathrm{T} 2 \mathrm{DM}^{(3)}$. Thus, a plant-based LC diet could offer significant advantages over an animal-based LC diet on cardiometabolic health.

The aims of the present study were two-fold. Firstly, the theoretical feasibility of a LC plant-based diet was determined by designing and calculating the cost of five weekly meal plans (two plant-based, and two animal-based) with two different carbohydrate thresholds $(50 \mathrm{~g} /$ day and $100 \mathrm{~g} /$ day) using Nutritics software. A low GI meal plan was created as a control. Secondly, the acceptability of a LC plant-based diet was determined through two focus group sessions with T2DM individuals $(n=7)$. Focus group questions were designed to explore the potential barriers of following a LC plant-based diet and capture viewpoints regarding the acceptability of the meal plans and opinions on cost and preparation time. Participants were given an exit questionnaire to summarise these views. Thematic analysis was carried out by the primary researcher to identify the key themes arising from the focus group transcript.

The plans created for this study successfully met the daily energy requirements (mean $=2080 \mathrm{kcal} /$ day), whilst not exceeding the set carbohydrates limits (50 g and 100g/day), thus a LC plant-based diet is feasible in relation to meeting the macronutrient targets of the meal plans. The focus groups revealed that all participants $(n=5 \mathrm{M} / 2 \mathrm{~F}$, aged $41-65 \mathrm{y})$ showed a willingness to follow a LC plant-based eating pattern, however, lacked confidence about sustaining this diet in the long term. Nearly all participants $(n=6)$ preferred the $100 \mathrm{~g}$ carbohydrate plant-based meal plan, in comparison to all other plans. Four dominant themes were derived from the transcript: 'Familiarity' and 'Convenience' are strong perceived barriers of plant-based and LC diets, participants are willing to reduce their intake of animal products, but not eliminate them completely, health and family eating habits are more important than cost, and participants have preconceived ideas about plant-based diets.

This study concluded that a LC plant-based diet is a potentially feasible dietary approach for T2DM management. Based on the participants' willingness to try a plant-based LC eating pattern, future human intervention studies should aim to determine if these diets are feasible in practice for both males and females with T2DM.

1. Kahleova H, Levin S \& Barnard N. (2017) Nutrients 9, 848.

2. Bolla AM, Caretto A, Laurenzi A et al. (2019) Nutrients 11, 962

3. Sajita A, Bhupathiraju SN, Rimm EB et al. (2016) PLoS Med 13, e1002039-e1002039 